\title{
Delay-dependent Stabilization Conditions of Controlled Positive Continuous-time Systems
}

\author{
Wafa Elloumi $^{1} \quad$ Abdellah Benzaouia $^{2} \quad$ Mohamed Chaabane $^{1}$ \\ ${ }^{1}$ Lab-STA, National School of Engineering of Sfax, University of Sfax PB 1173, 3038 Sfax, Tunisia \\ ${ }^{2}$ LAEPT URAC 28, University Cadi Ayyad, Faculty of Science Semlalia, BP 2390, Marrakech, Morocco
}

\begin{abstract}
The stabilization problem for a class of linear continuous-time systems with time-varying non differentiable delay is solved while imposing positivity in closed-loop. In particular, the synthesis of state-feedback controllers is studied by giving sufficient conditions in terms of linear matrix inequalities (LMIs). The obtained results are then extended to systems with non positive delay matrix by applying a memory controller. The effectiveness of the proposed method is shown by using numerical examples.
\end{abstract}

Keywords: Positive systems, delay systems, stabilization, linear matrix inequalities (LMIs), time-varying delay, non differentiable delay.

\section{Introduction}

This paper focuses on linear time-varying delay systems. The main objective is to build state-feedback controllers that make the closed-loop system with time-varying delay $\alpha$-exponentially stable and positive. This class of systems is frequently encountered in many fields of science and engineering, especially in biological modeling, economics, physiology and many others (see [1-4]). Since the existence of delay is generally a source of instability and degradation of performance, many researches and contributions have been done to analyze stability of linear systems with time-varying delay (see [5-8]).

In the literature, systems with nonnegative states are referred as positive systems (see [9-12] for general references). These systems appear in many practical problems, when the states represent physical quantities that have an intrinsically positive sign (absolute temperatures, levels, heights, concentrations, etc). The stabilization of systems with delays has been extensively studied in the literature (see [1318 ] and references therein), but only few authors have considered positive systems in this context of time-delay systems: we can quote $[11,14-16,19,20]$. In fact, all the latest references are only interested in asymptotic stability. While, in practice, it is judicious that the system can converge quickly (with a certain decay rate). It is well demonstrated that, unlike asymptotic stability of this class of systems, the exponential stability (with given decay rate) of positive time-varying and even constant delay systems is dependent on the magnitude of delays. Consequently, it is enough motivation to investigate the exponential stability of positive systems with delay.

For the stabilization purpose of systems with delays, some works on global stabilization via memoryless control laws have been proposed (see $[5,13]$ and references therein). On the other hand, other works were interested in the synthesis of controllers with memory as in $[15,18,21]$.

Generally, the time-varying delay is represented by a differentiable function as in [12] for positive systems. However,

Regular paper

Manuscript received August 22, 2013; accepted November 26, 2013 some new studies treat the case of non differentiable delays as $[22]$.

In this paper, the results of [8] based on the idea of [23], by considering a Lyapunov function composed of four terms, are extended to positive systems. The stabilization problem is then addressed for positive systems while correcting the proposed LMI in [23] which seems to need some rectifications. Thus, this work studies the problem of stabilization in the nonnegative state space for systems with varyingtime non differentiable delays. This problem, can be solved by using two types of control laws, memoryless and memory controllers to be able to apply the proposed technique to a large class of systems non initially positive and called controlled positive.

The remainder of the paper is structured as follows: Section 2 deals with the problem statement while some preliminary results are presented in Section 3. Section 4 presents the main results together with illustrative examples. Finally, Section 5 gives some concluding remarks.

Notations. $\mathbf{R}_{+}^{n}$ denotes the non-negative orthant of the $n$-dimensional real space $\mathbf{R}^{n}$.

$M^{\mathrm{T}}$ denotes the transpose of the real matrix $M$.

A matrix $M \in \mathbf{R}^{n \times n}$ is called a Metzler matrix if its off-diagonal elements are nonnegative. That is, if $M=$ $\left\{m_{i j}\right\}_{i, j=1}^{n}, M$ is Metzler if $m_{i j}>0$ when $i \neq j$.

A matrix $M \in \mathbf{R}^{n \times n}$ is called a $M$-matrix if and only if there exists a positive vector $\lambda$ such that $M \lambda>0$.

A matrix $M$ (or a vector) is said to be nonnegative if all its components are nonnegative (by notation $M \geqslant 0$ ). It is said to be positive if all its components are positive $(M>0)$.

A positive definite matrix $P \in \mathbf{R}^{n \times m}$ is denoted as $P>$ 0 .

A positive (nonnegative) vector $x \in \mathbf{R}^{n}$ is noted $x>$ $0(x \geqslant 0)$.

\section{Problem definition}

This paper deals with the following set of governed delayed linear systems: 


$$
\left\{\begin{array}{l}
\dot{x}(t)=A x(t)+A_{1} x(t-h(t))+B u(t), t \in \mathbf{R}_{+} \\
x(t)=\phi(t) \geqslant 0, \quad t \in\left[-h_{2}, 0\right] \\
A_{1} \geqslant 0
\end{array}\right.
$$

where $x \in \mathbf{R}^{n}$ is the state, $u \in \mathbf{R}^{m}$ is the control vector, and the matrices $A \in \mathbf{R}^{n \times n}, A_{1} \in \mathbf{R}^{n \times n}$ and $B \in \mathbf{R}^{n \times m}$ are supposed to be constant and known. $h(t) \in \mathbf{R}$ is the time-varying delay considered as a continuous function not necessarily differentiable, however bounded as

$$
0 \leqslant h_{1} \leqslant h(t) \leqslant h_{2} .
$$

Definition 1. Proposing two times $h_{a}=0.5\left(h_{2}+h_{1}\right)$, and $h_{r}=0.5\left(h_{2}-h_{1}\right)$, the time-varying delay $h(t)$ satisfying (2) can be expressed as

$$
h(t)=h_{a}+h_{r} \tau(t)
$$

where

$$
\tau(t)=\left\{\begin{array}{cc}
\frac{2 h(t)-\left(h_{2}+h_{1}\right)}{h_{2}-h_{1}}, & h_{2}>h_{1} \\
0, & h_{2}=h_{1} .
\end{array}\right.
$$

The main problem considered in this paper is the following: The state feedback control $u \in \mathbf{R}^{n \times m}$ is designed in two ways: firstly as a memoryless one and secondly as a memory control. In both cases, the controller must be designed in such a way that the resulting system in closed-loop is positive and $\alpha$-exponentially stable for any delay function $h(t)$ satisfying (2), no matter differentiable.

\section{Preliminaries}

In order to solve this problem, some useful results on delayed positive systems are now presented.

Define the following autonomous delayed continuoustime system:

$$
\left\{\begin{array}{l}
\dot{x}(t)=A x(t)+A_{1} x(t-h(t)) \\
x(t)=\phi(t) \geqslant 0, \quad t \in\left[-h_{2}, 0\right]
\end{array}\right.
$$

where matrix $A$ is Metzler and matrix $A_{1} \geqslant 0$.

Definition 2. Given any positive initial condition $x(t)=$ $\phi(t) \in \mathbf{R}_{+}^{n}, t \in\left[-h_{2}, 0\right]$, the delayed system (5) is said to be positive if the corresponding trajectory is never negative: $x(t) \in \mathbf{R}_{+}^{n}$ for all $t \geqslant 0$.

According to this definition, we need to find the condition under which the delayed system (1) is positive (see for example [19, 24]).

Lemma 1. System (5) is positive (i.e., $x(t) \in \mathbf{R}_{+}^{n}$ ) if and only if $A$ is a Metzler matrix and $A_{1}$ is a nonnegative matrix.

The following definition of $\alpha$-exponential stability is also recalled.

Definition 3. For given scalar $\alpha>0$, system (5) is called $\alpha$-exponentially stable if there exists a scalar $\delta>0$ such that the solution of system (5) satisfies $\|x(t)\| \leqslant \delta\|\phi\|_{c} \mathrm{e}^{-\alpha t}$, $\forall t>0$, where $\|\phi\|_{c}=\sup _{-\tau \leqslant s \leqslant 0}\|\phi(s)\|$.

The following results for positive systems are recalled for their use in the sequel.

Lemma 2. Matrix $M$ is Metzler if and only if there exists a positive scalar $\gamma$ such that: $M+\gamma I>0$.
Theorem $\mathbf{1}^{[25]}$. Let $M$ be a Metzler matrix. Then, $-M$ is an $M$-matrix if and only if there is a positive definite matrix $W$ such that matrix $M^{\mathrm{T}} W+W M$ is negative definite.

\section{Main results}

\subsection{Stability}

The following result presents a sufficient condition for the $\alpha$-exponential stability of the delayed system (5).

Theorem 2. For some given scalars $h_{1}, h_{2}, \alpha>$ 0 , the positive system (5) is $\alpha$-asymptotically stable for $h(t)$ satisfying (2), if there exist positive definite matrices $P, U, S, W, T_{i}$, matrices $M_{i},(i=1,2,3)$ with appropriate dimensions such that the following LMIs hold:

$$
\Omega=\left[\begin{array}{ccccc}
\Omega_{11} & \Omega_{12} & \Omega_{13} & 2 h_{a} M_{1}^{\mathrm{T}} & h_{r} T_{1}^{\mathrm{T}} A_{1} \\
* & \Omega_{22} & \Omega_{23} & 2 h_{a} M_{2}^{\mathrm{T}} & h_{r} T_{2}^{\mathrm{T}} A_{1} \\
* & * & \Omega_{33} & 2 h_{a} M_{3}^{\mathrm{T}} & h_{r} T_{3}^{\mathrm{T}} A_{1} \\
* & * & * & -2 h_{a} S & 0 \\
* & * & * & * & -h_{r} W
\end{array}\right]<0
$$

where

$$
\begin{aligned}
& \Omega_{11}=2 \alpha P+U+M_{1}^{\mathrm{T}}+M_{1}+T_{1}^{\mathrm{T}} A+A^{\mathrm{T}} T_{1} \\
& \Omega_{12}=-M_{2}+M_{1}^{\mathrm{T}}+T_{1}^{\mathrm{T}} A_{1}+A^{\mathrm{T}} T_{2} \\
& \Omega_{13}=P+M_{3}+M_{1}^{\mathrm{T}}-T_{1}^{\mathrm{T}}+A^{\mathrm{T}} T_{3} \\
& \Omega_{22}=-\mathrm{e}^{-2 \alpha h_{a}} U-M_{2}-M_{2}^{\mathrm{T}}+T_{2}^{\mathrm{T}} A_{1} X+A_{1}^{\mathrm{T}} T_{2} \\
& \Omega_{23}=-M_{3}-T_{2}^{\mathrm{T}}+A_{1}^{\mathrm{T}} T_{3} \\
& \Omega_{33}=h_{a} S+h_{r} W-T_{3}^{\mathrm{T}}-T_{3} .
\end{aligned}
$$

Proof. See the Appendix.

\subsection{Stabilization by memoryless controller}

This section presents the main results. First, the stabilization problem for general systems is studied and solved.

In other words, the main problem reduces to looking for a memoryless state feedback law $u(t)=K x(t)$, leading to the delayed closed-loop system defined by

$$
\left\{\begin{array}{l}
\dot{x}(t)=(A+B K) x(t)+A_{1} x(t-h(t)) \\
x(t)=\phi(t) \geqslant 0, \quad t \in\left[-h_{2}, 0\right]
\end{array}\right.
$$

where matrix $K \in \mathbf{R}^{m \times n}$ has to be selected to ensure the following problem:

Find sufficient conditions on matrices $A, A_{1} \in \mathbf{R}^{n \times n}$, $B \in \mathbf{R}^{n \times m}$, such that there exists a matrix $K \in \mathbf{R}^{m \times n}$ satisfying:

1) Positivity in closed-loop $\left(A_{c}=A+B K\right.$ is a Metzler matrix).

2) Closed-loop $\alpha$-exponential stability.

Note that no assumption on matrix $A$ to be Metzler is needed.

Theorem 3. For some given scalars $h_{1}, h_{2}, \alpha>0$, $\gamma>0$, system (8) is $\alpha$-exponentially stable and positive for $h(t)$ satisfying (2), if there exist positive definite matrices $P, U, S, W$, diagonal positive matrices $X_{i}$, matrices $\tilde{M}_{i},(i=$ $1,2,3)$ and $Y_{i}$ with appropriate dimensions such that the 
following LMIs hold:

$$
\left[\begin{array}{ccccc}
\hat{\Omega}_{11} & \hat{\Omega}_{12} & \hat{\Omega}_{13} & 2 h_{a} \hat{M}_{1}^{\mathrm{T}} & h_{r} A_{1} X_{1} \\
* & \hat{\Omega}_{22} & \hat{\Omega}_{23} & 2 h_{a} \hat{M}_{2}^{\mathrm{T}} & h_{r} A_{1} X_{2} \\
* & * & \hat{\Omega}_{33} & 2 h_{a} \hat{M}_{3}^{\mathrm{T}} & h_{r} A_{1} X_{3} \\
* & * & * & -2 h_{a} \hat{S} & 0 \\
* & * & * & * & -h_{r} \hat{W}
\end{array}\right]<0
$$$$
A X+B Y+\gamma X>0
$$

with $T_{i}=\rho_{i} X, K=Y X^{-1}$ and

$$
\begin{aligned}
\hat{\Omega}_{11}= & 2 \alpha \hat{P}+\hat{U}+\hat{M}_{1}^{\mathrm{T}}+\hat{M}_{1}+A X_{1}+ \\
& B Y_{1}+X_{1} A^{\mathrm{T}}+Y_{i}^{\mathrm{T}} B^{\mathrm{T}} \\
\hat{\Omega}_{12}= & -\hat{M}_{2}+\hat{M}_{1}^{\mathrm{T}}+A_{1} X_{1}+\rho_{2} A^{\mathrm{T}}+\rho_{2} Y^{\mathrm{T}} B^{\mathrm{T}} \\
\hat{\Omega}_{13}= & \hat{P}+\hat{M}_{3}+\hat{M}_{1}^{\mathrm{T}}-\rho_{1} X+\rho_{3} X A^{\mathrm{T}}+\rho_{3} Y^{\mathrm{T}} B^{\mathrm{T}} \\
\hat{\Omega}_{22}= & -\mathrm{e}^{-2 \alpha h_{a}} \hat{U}-\hat{M}_{2}+\hat{M}_{2}^{\mathrm{T}}+\rho_{2} A_{1} X+\rho_{2} X A_{1}^{\mathrm{T}} \\
\hat{\Omega}_{23}= & -\hat{M}_{3}-\rho_{2} X+\rho_{3} X A_{1}^{\mathrm{T}} \\
\hat{\Omega}_{33}= & h_{a} \hat{S}+h_{r} \hat{W}-2 \rho_{3} X .
\end{aligned}
$$

Proof. We begin by the proof of the $\alpha$-exponential stability.

Pre- and post-multiply LMI (6), where matrix $A$ is replaced by $A+B K$, by $\hat{X}=\operatorname{diag}\{X, X, X, X, X\}$. Let $T_{i}=\rho_{i} X$, one obtains

$$
\begin{aligned}
X \Omega_{11} X= & 2 \alpha X P X+X U X+X M_{1}^{\mathrm{T}} X+X M_{1} X+ \\
& \rho_{1}(A+B K) X+\rho_{1} X(A+B K)^{\mathrm{T}} \\
X \Omega_{12} X= & -X M_{2} X+X M_{1}^{\mathrm{T}} X+ \\
& \rho_{1} A_{1} X+\rho_{2} X(A+B K)^{\mathrm{T}} \\
X \Omega_{13} X= & X P X+X M_{3} X+X M_{1}^{\mathrm{T}} X- \\
& \rho_{1} X+\rho_{3} X(A+B K)^{\mathrm{T}} \\
X \Omega_{14} X= & 2 h_{a} X M_{1}^{\mathrm{T}} X \\
X \Omega_{15} X= & h_{r} \rho_{1} A_{1} X \\
X \Omega_{22} X= & -\mathrm{e}^{-2 \alpha h_{a}} X U X-X M_{2} X+X M_{2}^{\mathrm{T}} X+ \\
& \rho_{2} A_{1} X+\rho_{2} X A_{1}^{\mathrm{T}} \\
X \Omega_{23} X= & -X M_{3} X-\rho_{2} X+\rho_{3} X A_{1}^{\mathrm{T}} \\
X \Omega_{33} X= & h_{a} X S X+h_{r} X W X-2 \rho_{3} X \\
X \Omega_{44} X= & -2 h_{a} X S X \\
X \Omega_{55} X= & -h_{r} X W X .
\end{aligned}
$$

Thus, we replace $X \Omega_{i j} X, i, j=1, \cdots, 5$ by $\hat{\Omega}_{i j}$. The same treatment is done to matrices $P, U, S, W$, and $M_{i}, i=$ $1, \cdots, 3$. Finally, letting $K=Y X^{-1}$, we obtain $\phi=$ $\hat{X} \Omega \hat{X}<0$, where $\hat{X}=\operatorname{diag}\{X, X, X, X, X\}$. Hence, the resulting closed-loop system (8) is $\alpha$-exponentially stable with the feedback control $u(t)=K x(t)$.

To complete the proof, one has to show the positivity condition (10) by using the idea of Theorem 1 . Let condition (10) be satisfied. Since matrix $X$ is diagonal positive, $X^{-1}$ is also diagonal positive. By post-multiplying (10) by $X^{-1}$, one obtains $A+B Y X^{-1}+\gamma I>0$, that is $A+B K+\gamma I>0$, for $\gamma>0$, according to Lemma 2, the matrix in closed-loop $A+B K$ is Metzler while matrix $A_{1}$ is assumed to be non negative. The system in closed-loop, which is $\alpha$-exponentially stable, is then positive.
Remark 1. It is worth noting that in [23], only matrices $A+B K$ are pre and post multiplied by $X$ to have $X \Omega_{11} X=$ $2 \alpha P+U+M_{1}^{\mathrm{T}}+M_{1}+\rho_{1}(A+B K) X+\rho_{1} X(A+B K)^{\mathrm{T}}$, which is obviously wrong.

\subsection{Stabilization by memory controller}

This section deals with memory controller to avoid any assumption of positivity on the delay matrix $A_{1}$. For this, consider the following control law:

$$
u(t)=K x(t)+F x(t-h(t)) .
$$

The system in closed-loop becomes

$$
\left\{\begin{array}{l}
\dot{x}(t)=(A+B K) x(t)+\left(A_{1}+B F\right) x(t-h(t)) \\
x(t)=\phi(t) \geqslant 0, \quad t \in\left[-h_{2}, 0\right]
\end{array}\right.
$$

where both matrices $K, F \in \mathbf{R}^{m \times n}$ are selected to ensure that this closed-loop system is simultaneously positive and $\alpha$-exponentially stable. In other words, find sufficient conditions on matrices $A, A_{1} \in \mathbf{R}^{n \times n}, B \in \mathbf{R}^{n \times m}, K, F \in \mathbf{R}^{m \times n}$ satisfying:

1) Positivity in closed-loop $\left(A_{c}=A+B K\right.$ is a Metzler matrix and $A_{1 c}=A_{1}+B F$ is non-negative).

2) Closed-loop $\alpha$-exponential stability.

Note that assumptions on matrices $A, A_{1}$ are no more needed.

Theorem 4. For some given scalars $h_{1}, h_{2}, \alpha>0$, $\gamma>0$, system (13) is $\alpha$-exponentially stable and positive for $h(t)$ satisfying $(2)$ if there exist positive definite matrices $P, U, S, W$, diagonal positive matrix $X$, matrices $\hat{M}_{i}, \quad(i=$ $1,2,3), Y$ and $Z$ with appropriate dimensions such that the following LMIs hold:

$$
\begin{aligned}
& \Psi<0 \\
& A X+B Y+\gamma X>0 \\
& A_{1} X+B Z \geqslant 0
\end{aligned}
$$

with $T_{i}=\rho_{i} X, K=Y X^{-1}, F=Z X^{-1}$ and

$$
\Psi=\left[\begin{array}{ccccc}
\tilde{\Omega}_{11} & \tilde{\Omega}_{12} & \tilde{\Omega}_{13} & 2 h_{a} \tilde{M}_{1}^{\mathrm{T}} & \rho_{1} h_{r} A_{1} X+\rho_{1} h_{r} B Z \\
* & \tilde{\Omega}_{22} & \tilde{\Omega}_{23} & 2 h_{a} \tilde{M}_{2}^{\mathrm{T}} & \rho_{2} h_{r} A_{1} X+\rho_{2} h_{r} B Z \\
* & * & \tilde{\Omega}_{33} & 2 h_{a} \tilde{M}_{3}^{\mathrm{T}} & \rho_{3} h_{r} A_{1} X+\rho_{3} h_{r} B Z \\
* & * & * & -2 h_{a} \tilde{S} & 0 \\
* & * & * & * & -h_{r} \tilde{W}
\end{array}\right]
$$

and

$$
\begin{aligned}
\tilde{\Omega}_{11}= & 2 \alpha \tilde{P}+\tilde{U}+\tilde{M}_{1}^{\mathrm{T}}+\tilde{M}_{1}+\rho_{1} A X+ \\
& \rho_{1} B Y+\rho_{1} X A^{T}+\rho_{1} Y^{\mathrm{T}} B^{\mathrm{T}} \\
\tilde{\Omega}_{12}= & -\tilde{M}_{2}+\tilde{M}_{1}^{\mathrm{T}}+\rho_{1} A_{1} X+ \\
& \rho_{1} B Z+\rho_{2} A^{\mathrm{T}}+\rho_{2} Y^{\mathrm{T}} B^{\mathrm{T}} \\
\tilde{\Omega}_{13}= & \tilde{P}+\tilde{M}_{3}+\tilde{M}_{1}^{\mathrm{T}}-\rho_{1} X+ \\
& \rho_{3} X A^{\mathrm{T}}+\rho_{3} Y^{\mathrm{T}} B^{\mathrm{T}}
\end{aligned}
$$




$$
\begin{aligned}
\tilde{\Omega}_{22}= & -\mathrm{e}^{-2 \alpha h_{a}} \tilde{U}-\tilde{M}_{2}+\tilde{M}_{2}^{\mathrm{T}}+ \\
& \rho_{2} A_{1} X+\rho_{2} B Z+\rho_{2} X A_{1}^{\mathrm{T}}+ \\
& \rho_{2} Z^{\mathrm{T}} B^{\mathrm{T}} \\
\tilde{\Omega}_{23}= & -\tilde{M}_{3}-\rho_{2} X+\rho_{3} X A_{1}^{\mathrm{T}}+\rho_{3} Z^{\mathrm{T}} B^{\mathrm{T}} \\
\tilde{\Omega}_{33}= & h_{a} \tilde{S}+h_{r} \tilde{W}-2 \rho_{3} X .
\end{aligned}
$$

Proof. We begin by the proof of the $\alpha$-exponential stability. Pre- and post-multiply LMI (6), where matrix $A$ is replaced by $A+B K$ and matrix $A_{1}$ by $A_{1}+B F$, by $\hat{X}=\operatorname{diag}\{X, X, X, X, X\}$. Let $T_{i}=\rho_{i} X$, one obtains

$$
\begin{aligned}
X \Omega_{11} X= & 2 \alpha X P X+X U X+X M_{1}^{\mathrm{T}} X+X M_{1} X+ \\
& \rho_{1}(A+B K) X+\rho_{1} X(A+B K)^{\mathrm{T}} \\
X \Omega_{12} X= & -X M_{2} X+X M_{1}^{\mathrm{T}} X+\rho_{1}\left(A_{1}+B F\right) X+ \\
& \rho_{2} X(A+B K)^{\mathrm{T}} \\
X \Omega_{13} X= & X P X+X M_{3} X+X M_{1}^{\mathrm{T}} X-\rho_{1} X+ \\
& \rho_{3} X(A+B K)^{\mathrm{T}} \\
X \Omega_{14} X= & 2 h_{a} X M_{1}^{\mathrm{T}} X \\
X \Omega_{15} X= & h_{r} \rho_{1} A_{1} X \\
X \Omega_{22} X= & -\mathrm{e}^{-2 \alpha h_{a}} X U X-X M_{2} X+X M_{2}^{\mathrm{T}} X+ \\
& \rho_{2}\left(A_{1}+B F\right) X+\rho_{2} X\left(A_{1}+B F\right)^{\mathrm{T}} \\
X \Omega_{23} X= & -X M_{3} X-\rho_{2} X+\rho_{3} X\left(A_{1}+B F\right)^{\mathrm{T}} \\
X \Omega_{33} X= & h_{a} X S X+h_{r} X W X-2 \rho_{3} X \\
X \Omega_{44} X= & -2 h_{a} X S X \\
X \Omega_{55} X= & -h_{r} X W X .
\end{aligned}
$$

Thus, we replace $X \Omega_{i j} X, i, j=1, \cdots, 5$, by $\tilde{\Omega}_{i j}$. The same treatment is done to matrices $P, U, S, W$ and $M_{i}, i=$ $1, \cdots, 3$. Finally, letting $K=Y X^{-1}$ and $F=Z X^{-1}$, we obtain $\hat{X} \Omega \hat{X}<0$, where $\hat{X}=\operatorname{diag}\{X, X, X, X, X\}$. Hence, the resulting closed-loop system (8) is $\alpha$-exponentially stable with the feedback control $u(t)=K x(t)+F x(t-h(t))$.

To complete the proof, one has to show the positivity conditions (15) and (16) by using the idea of Theorem 1. Let condition (15) and (16) be satisfied. Since matrix $X$ is diagonal positive, $X^{-1}$ is also diagonal positive. By post-multiplying (15) and (16) by $X^{-1}$, one obtains $A+B Y X^{-1}+\gamma \mathbb{I}>0$ and $A_{1}+B Z X^{-1}$ respectively, that is $A+B K+\gamma \mathbb{I}>0$, for $\gamma>0$, according to Lemma 2, the matrix in closed-loop $A_{c}=A+B K$ is Metzler while matrix $A_{1 c}=A_{1}+B F$ is non negative. The system in closed-loop which is $\alpha$-exponentially stable is then positive.

\section{$5 \quad$ Examples}

In this section, two examples are studied, one for memoryless controller and one for controller with memory.

Example 1. Memoryless state-feedback stabilization. Consider a delayed system described by (1), with the following system matrices:

$$
A=\left[\begin{array}{ll}
-1 & -0.5 \\
-3 & -0.7
\end{array}\right], A_{1}=\left[\begin{array}{ll}
0.1 & 0.2 \\
0.3 & 0.1
\end{array}\right]
$$

$$
B=\left[\begin{array}{cc}
-0.4 & 0 \\
0 & -0.2
\end{array}\right] \text {. }
$$

In order to show the effectiveness of our results, two types of delay functions, differentiable and not differentiable, are used to plot the evolution of the system trajectories.

The first delay function is given by

$$
h(t)=\frac{0.5}{1+t}
$$

The second delay function is given by

$$
h(t)= \begin{cases}0.1+0.4 \sin ^{2}(t), & \text { if } t \in[0, \pi] \\ 0.5, & \text { elsewhere. }\end{cases}
$$

It can be seen that the open-loop system is not positive (although $A_{1}$ is nonnegative, there are off-diagonal negative elements in $A$ ). The objective is to design a state feedback controller $u=K x$ that stabilizes the system and makes the closed-loop states nonnegative for any value of the delay $h(t)$ (starting from any nonnegative initial condition). For this, conditions of Theorem 3 must be fulfilled. The gain of a stabilizing control is given by any feasible solution to the above LMIs. It is very important to notice that we should choose $T_{i}, i=1, \cdots, 3$ with different values to obtain the feasibility of this example. In other words, we should have $T_{1} \neq T_{2} \neq T_{3}$ and likewise. Otherwise, the LMIs may not be feasible.

For this example, the LMIs are feasible and their solutions are given for $\gamma=20, \alpha=0.2, \rho_{1}=0.9, \rho_{2}=0.5, \rho_{3}=$ 0.4 , by

$$
\begin{aligned}
& K=\left[\begin{array}{cc}
18.0074 & -1.4762 \\
-15.1436 & 37.4535
\end{array}\right] \\
& A_{c}=\left[\begin{array}{cc}
-8.2029 & 0.0905 \\
0.0287 & -8.1907
\end{array}\right] \\
& X=\left[\begin{array}{cc}
0.5757 & 0 \\
0 & 0.5776
\end{array}\right] \\
& P=\left[\begin{array}{cc}
1.3700 & 0.0063 \\
0.0063 & 1.3701
\end{array}\right] .
\end{aligned}
$$

It can be seen that with this controller, the feedback system is positive and the state evolution for the system remains always within the nonnegative orthant. For example, the state trajectories from several different initial positive conditions can be seen as follows: Fig. 1 for differentiable delay (19), and Fig. 2 for non differentiable delay (20) which is presented in Fig. 3. 


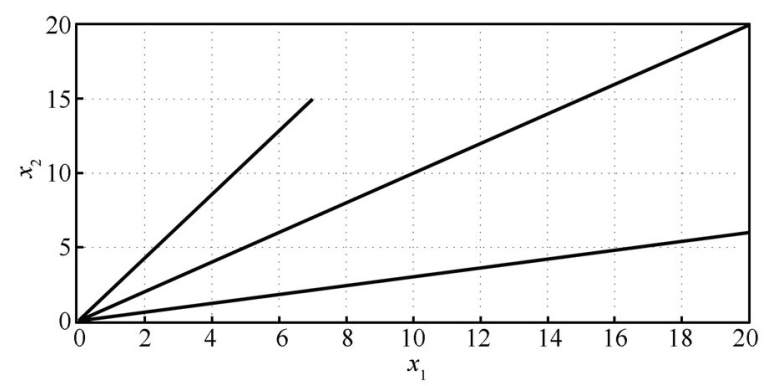

Fig. 1 Trajectories of the states from different initial values obtained with delay function (19)

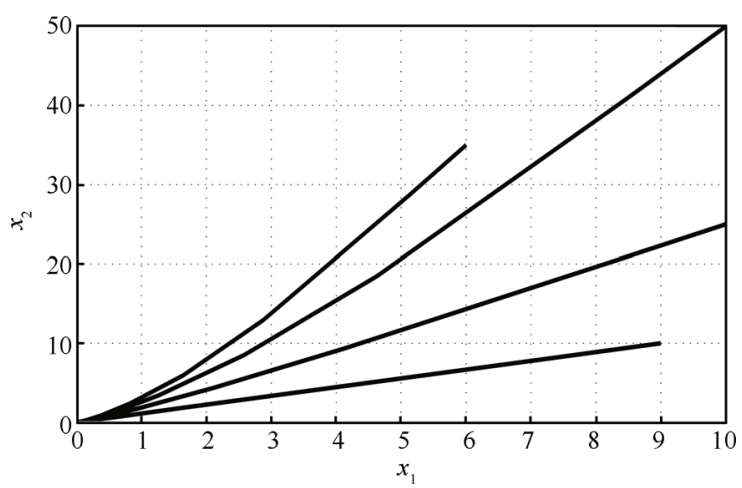

Fig. 2 Trajectories of the states from different initial values obtained with delay function (20)

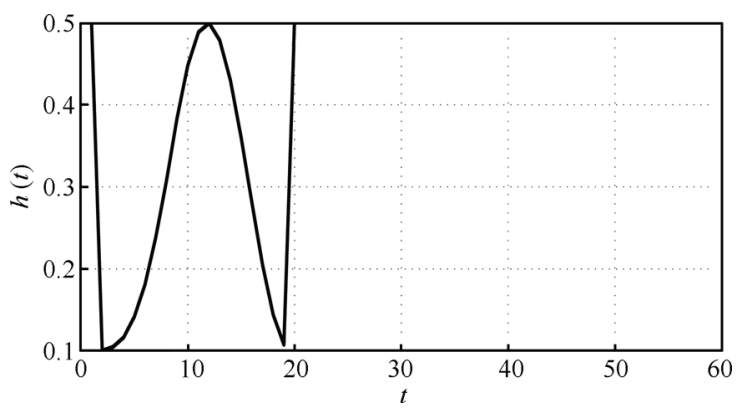

Fig. 3 Trajectory of the non differentiable time-varying delay (20)

Example 2. Memory state-feedback stabilization. Consider a delayed system described by (1), with the following system matrices:

$$
\begin{aligned}
& A=\left[\begin{array}{ll}
-1 & -0.5 \\
-3 & -0.7
\end{array}\right], A_{1}=\left[\begin{array}{cc}
-0.1 & -0.2 \\
0.3 & -0.1
\end{array}\right], \\
& B=\left[\begin{array}{cc}
-0.5 & 0 \\
0 & -0.2
\end{array}\right] .
\end{aligned}
$$

In order to show the effectiveness of our results, the same delay functions (19) and (20) are considered.

It can be seen that the open-loop system is not positive (although $A_{1}$ is nonnegative, there are off-diagonal negative elements in $A$ ). The objective is to design a memory state feedback controller $u=K x+F x(t-h(t))$ that stabilizes the system and makes the closed-loop states nonnegative for any value of the delay $h(t)$ (starting from any nonnegative initial condition). For this, conditions of Theorem 4 must be fulfilled. The gain of the stabilizing controller is given by any feasible solution to the above LMIs. It is very important to notice that we should choose $T_{i}, i=1, \cdots, 3$ with different values to obtain the feasibility of this example. In other words, we should choose $T_{1} \neq T_{2} \neq T_{3}$.

For this example, the LMIs are feasible with $\gamma=20, \alpha=$ $0.2, \rho_{1}=0.9, \rho_{2}=0.5, \rho_{3}=0.4$. The results are given by

$$
\begin{aligned}
& K=\left[\begin{array}{cc}
17.3689 & -1 \\
-15 & 44.9221
\end{array}\right] \\
& F=\left[\begin{array}{cc}
-2.7447 & -0.4 \\
1.5 & -6.8617
\end{array}\right] \\
& A_{c}=\left[\begin{array}{cc}
-9.6844 & 0 \\
0 & -9.6844
\end{array}\right] \\
& A_{1 c}=\left[\begin{array}{cc}
1.2723 & 0 \\
0 & 1.2723
\end{array}\right] \\
& X=\left[\begin{array}{cc}
230.7098 & 0 \\
0 & 230.7098
\end{array}\right] \\
& P=\left[\begin{array}{cc}
696.7876 & 0 \\
0 & 696.7876
\end{array}\right] .
\end{aligned}
$$

It can be seen that with this controller, the feedback system is positive and the state evolution of the system remains always within the nonnegative orthant. For example, the state trajectories from several initial positive conditions can be seen in Fig. 4 for differentiable delay (19) and Fig. 5 for non differentiable delay (20).

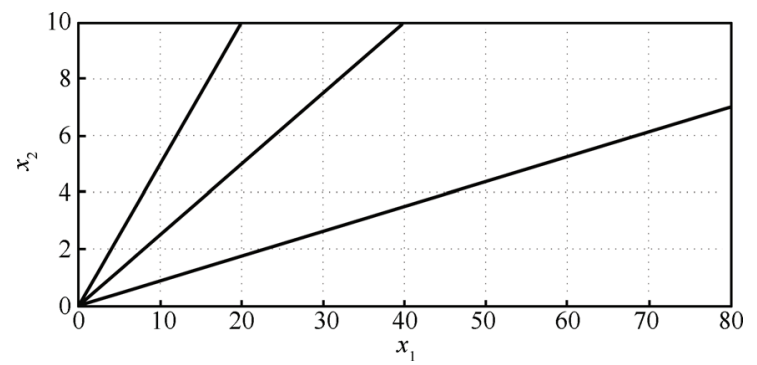

Fig. 4 Trajectories of the states from different initial values obtained with delay function (19)

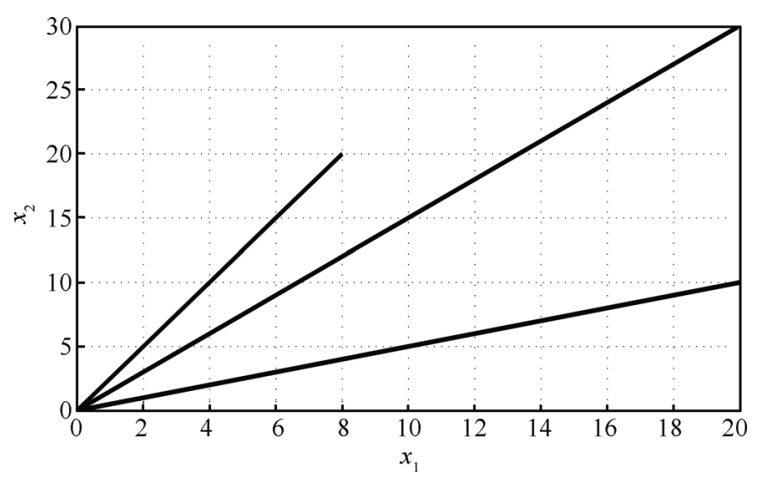

Fig. 5 Trajectories of the states from different initial values obtained with delay function (20) 


\section{Conclusions}

This paper has solved the problem of imposing non negativity to closed-loop states using state feedback control for continuous-time systems with non differentiable timevarying delay. First, sufficient conditions of stability for the system, by using LMIs, have been proposed. The same idea was then followed to solve the problem of stabilization while guaranteeing positivity of the state even for non positive systems in open-loop. The obtained results are then extended to systems with non positive delay matrix by applying a memory controller. Some examples have been presented to illustrate the proposed approach, showing its feasibility and simplicity.

\section{Appendix}

Proof of Theorem 2. The proof follows the same arguments as in the work of [23] without constant delay, but with additional slack variables as shown in [8] for the $\alpha$ exponential stability part. It is worth noting that for stability purpose, matrix $T_{1}$ is not necessarily diagonal as suggested by Theorem 1 .

For the proof, the following Lyapunov-Krasovskii function was used as in [8]:

$$
V(x(t))=\sum_{i=1}^{4} V_{i}(t)
$$

where

$$
\begin{aligned}
& V_{1}(t)=\mathrm{e}^{2 \alpha t} x^{\mathrm{T}}(t) P x(t) \\
& V_{2}(t)=\int_{t-h_{a}}^{\mathrm{T}} \mathrm{e}^{2 \alpha t} x^{\mathrm{T}}(s) U x(s) \mathrm{d} s \\
& V_{3}(t)=\int_{-h_{a}}^{t-h_{a}} \int_{t+s}^{\mathrm{T}} \mathrm{e}^{2 \alpha t} \dot{x}(\theta)^{\mathrm{T}}(s) S \dot{\theta}(s) \mathrm{d} \theta \mathrm{d} s \\
& V_{4}(t)=\int_{-h_{a}}^{-h_{1}} \int_{t+s}^{\mathrm{T}} \mathrm{e}^{2 \alpha t} \dot{x}(\theta)^{\mathrm{T}}(s) W \dot{\theta}(s) \mathrm{d} \theta \mathrm{d} s .
\end{aligned}
$$

By derivating these functionals, we obtain

$$
\dot{V}(x(t))=\sum_{i=1}^{3} \dot{V}_{i}(t)
$$

where

$$
\begin{aligned}
& \dot{V}_{1}(t)= 2 \alpha \mathrm{e}^{2 \alpha t} x^{\mathrm{T}}(t) P x(t)+2 \mathrm{e}^{2 \alpha t} x^{\mathrm{T}}(t) P \dot{x}(t) \\
& \dot{V}_{2}(t)= \mathrm{e}^{2 \alpha t}\left[x^{\mathrm{T}}(t) U x(t)-\mathrm{e}^{-2 \alpha h_{a}} x^{\mathrm{T}}\left(t-h_{a}\right) U x\left(t-h_{a}\right)\right] \\
& \dot{V}_{3}(t)= \mathrm{e}^{2 \alpha t}\left[\dot{x}^{\mathrm{T}}(t) h_{a} S \dot{x}(t)-\int_{t-h_{a}}^{\mathrm{T}} \mathrm{e}^{-2 \alpha(s-t)} \dot{x}^{\mathrm{T}}(s) S \dot{x}(s) \mathrm{d} s\right] \\
& \dot{V}_{4}(t)= \mathrm{e}^{2 \alpha t}\left[\dot{x}^{\mathrm{T}}(t) h_{r} W \dot{x}(t)-\int_{t-h_{a}}^{t-h_{1}} \mathrm{e}^{-2 \alpha(s-t)} \times\right. \\
&\left.\dot{x}^{\mathrm{T}}(s) W \dot{x}(s) \mathrm{d} s\right] .
\end{aligned}
$$

Certainly, for any scalar $s \in\left[t-h_{a}, t\right]$, we have

$$
\mathrm{e}^{-2 \alpha h_{a}} \leqslant \mathrm{e}^{-2 \alpha(s-t)} \leqslant 1
$$

and

$$
-\int_{t-h_{a}}^{\mathrm{T}} \mathrm{e}^{-2 \alpha(s-t)} \dot{x}^{\mathrm{T}}(s) S \dot{x}(s) \mathrm{d} s \leqslant
$$

$$
-\mathrm{e}^{-2 \alpha h_{a}} \int_{t-h_{a}}^{\mathrm{T}} \dot{x}^{\mathrm{T}}(s) S \dot{x}(s) \mathrm{d} s
$$

For any scalar $s \in\left[t-h_{a}, t-h_{r}\right]$, we have

$$
\mathrm{e}^{-2 \alpha h_{r}} \leqslant \mathrm{e}^{-2 \alpha(s-t)} \leqslant 1
$$

and

$$
\begin{aligned}
-\int_{t-h_{a}}^{t-h_{1}} \mathrm{e}^{-2 \alpha(s-t)} \dot{x}^{\mathrm{T}}(s) W \dot{x}(s) \mathrm{d} s \leqslant \\
-\mathrm{e}^{-2 \alpha h_{r}} \int_{t-h_{a}}^{t-h_{1}} \dot{x}^{\mathrm{T}}(s) W \dot{x}(s) \mathrm{d} s \leqslant \\
-\mathrm{e}^{-2 \alpha h_{r}} \int_{t-h_{a}}^{t-h(t)} \dot{x}^{\mathrm{T}}(s) W \dot{x}(s) \mathrm{d} s .
\end{aligned}
$$

Using (A8) and (A9), we obtain

$$
\begin{aligned}
\dot{V}(x(t)) \leqslant & 2 \alpha \mathrm{e}^{2 \alpha t} x^{\mathrm{T}}(t) P x(t)+2 \mathrm{e}^{2 \alpha t} x^{\mathrm{T}}(t) P \dot{x}(t)+ \\
& \mathrm{e}^{2 \alpha t}\left[x^{\mathrm{T}}(t) U x(t)-\mathrm{e}^{-2 \alpha h_{a}} x^{\mathrm{T}}\left(t-h_{a}\right) \times\right. \\
& \left.U x\left(t-h_{a}\right)\right]+\mathrm{e}^{2 \alpha t}\left[\dot{x}^{\mathrm{T}}(t) h_{a} S \dot{x}(t)-\right. \\
& \left.\mathrm{e}^{-2 \alpha h_{a}} \int_{t-h_{a}}^{\mathrm{T}} \dot{x}^{\mathrm{T}}(s) S \dot{x}(s) \mathrm{d} s\right]+ \\
& \mathrm{e}^{2 \alpha t}\left[\dot{x}^{\mathrm{T}}(t) h_{r} W \dot{x}(t)-\right. \\
& \left.\mathrm{e}^{-2 \alpha h_{r}} \int_{t-h_{a}}^{t-h(t)} \dot{x}^{\mathrm{T}}(s) W \dot{x}(s) \mathrm{d} s\right]= \\
& \mathrm{e}^{2 \alpha t}\left[x^{\mathrm{T}}(t)(2 \alpha P+U) x(t)+2 x^{\mathrm{T}}(t) P \dot{x}(t)+\right. \\
& \dot{x}^{\mathrm{T}}(t)\left(h_{a} S+h_{r} W\right) \dot{x}(t)- \\
& \mathrm{e}^{-2 \alpha h_{a}} x^{\mathrm{T}}\left(t-h_{a}\right) U x\left(t-h_{a}\right)- \\
& \mathrm{e}^{-2 \alpha h_{a}} \int_{t-h_{a}}^{\mathrm{T}} \dot{x}^{\mathrm{T}}(s) S \dot{x}(s) \mathrm{d} s- \\
& \left.\mathrm{e}^{-2 \alpha h_{r}} \int_{t-h_{a}}^{t-h_{(t)}} \dot{x}^{\mathrm{T}}(s) W \dot{x}(s) \mathrm{d} s\right] .
\end{aligned}
$$

Expanding the Newton-Leibniz formula, we have

$$
x(t)-x\left(t-h_{a}\right)-\int_{t-h_{a}}^{\mathrm{T}} \dot{x}(s) \mathrm{d} s=0 .
$$

Thus, by introducing the matrices $M_{i}$ and $T_{i}, i=1,2,3$, we obtain the following equalities:

$$
\begin{gathered}
2 \mathrm{e}^{2 \alpha t}\left[x^{\mathrm{T}}(t) M_{1}^{\mathrm{T}}+x^{\mathrm{T}}\left(t-h_{a}\right) M_{2}^{\mathrm{T}}+\dot{x}^{\mathrm{T}}(t) M_{3}^{\mathrm{T}}\right] \times \\
{\left[x(t)-x\left(t-h_{a}\right)-\int_{t-h_{a}}^{\mathrm{T}} \dot{x}(s) \mathrm{d} s\right]=0}
\end{gathered}
$$

and

$$
\begin{array}{r}
2 \mathrm{e}^{2 \alpha t}\left[x^{\mathrm{T}}(t) T_{1}^{\mathrm{T}}+x^{\mathrm{T}}\left(t-h_{a}\right) T_{2}^{\mathrm{T}}+\dot{x}^{\mathrm{T}}(t) T_{3}^{\mathrm{T}}\right] \times[A x(t)- \\
\left.B x\left(t-h_{a}\right)-B \int_{t-h_{a}}^{\mathrm{T}} \dot{x}(s) \mathrm{d} s-B \dot{x}(t)\right]=0 .
\end{array}
$$


Adding (A12) and (A13) to (A10), we obtain

$$
\begin{aligned}
\dot{V}(x(t)) \leqslant & 2 \alpha \mathrm{e}^{2 \alpha t} x^{\mathrm{T}}(t) P x(t)+2 \mathrm{e}^{2 \alpha t} x^{\mathrm{T}}(t) P \dot{x}(t)+ \\
& \mathrm{e}^{2 \alpha t}\left[x^{\mathrm{T}}(t) U x(t)-\mathrm{e}^{-2 \alpha h_{a}} x^{\mathrm{T}}\left(t-h_{a}\right) \times\right. \\
& \left.U x\left(t-h_{a}\right)\right]+\mathrm{e}^{2 \alpha t}\left[\dot{x}^{\mathrm{T}}(t) h_{a} S \dot{x}(t)-\right. \\
& \left.\mathrm{e}^{-2 \alpha h_{a}} \int_{t-h_{a}}^{\mathrm{T}} \dot{x}^{\mathrm{T}}(s) S \dot{x}(s) \mathrm{d} s\right]+ \\
& \mathrm{e}^{2 \alpha t}\left[\dot{x}^{\mathrm{T}}(t) h_{r} W \dot{x}(t)-\right. \\
& \left.\mathrm{e}^{-2 \alpha h_{r}} \int_{t-h_{a}}^{t-h(t)} \dot{x}^{\mathrm{T}}(s) W \dot{x}(s) \mathrm{d} s\right]= \\
& \mathrm{e}^{2 \alpha t}\left[x^{\mathrm{T}}(t)(2 \alpha P+U) x(t)+2 x^{\mathrm{T}}(t) P \dot{x}(t)+\right. \\
& \dot{x}^{\mathrm{T}}(t)\left(h_{a} S+h_{r} W\right) \dot{x}(t)- \\
& \mathrm{e}^{-2 \alpha h_{a}} x^{\mathrm{T}}\left(t-h_{a}\right) U x\left(t-h_{a}\right)- \\
& \mathrm{e}^{-2 \alpha h_{a}} \int_{t-h_{a}}^{\mathrm{T}} \dot{x}^{\mathrm{T}}(s) S \dot{x}(s) \mathrm{d} s- \\
& \left.\mathrm{e}^{-2 \alpha h_{r}} \int_{t-h_{a}}^{t-h(t)} \dot{x}^{\mathrm{T}}(s) W \dot{x}(s) d s\right]+ \\
& 2 \mathrm{e}^{2 \alpha t}\left[x^{\mathrm{T}}(t) M_{1}^{\mathrm{T}}+x^{\mathrm{T}}\left(t-h_{a}\right) M_{2}^{\mathrm{T}}+\dot{x}^{\mathrm{T}}(t) M_{3}^{\mathrm{T}}\right] \times \\
& {\left[x(t)-x\left(t-h_{a}\right)-\int_{t-h_{a}}^{\mathrm{T}} \dot{x}(s) \mathrm{d} s\right]+} \\
& 2 \mathrm{e}^{2 \alpha t}\left[x^{\mathrm{T}}(t) T_{1}^{\mathrm{T}}+x^{\mathrm{T}}\left(t-h_{a}\right) T_{2}^{\mathrm{T}}+\dot{x}^{\mathrm{T}}(t) T_{3}^{\mathrm{T}}\right] \times \\
& {\left[A x(t)-B x\left(t-h_{a}\right)-B \int_{a}^{\mathrm{T}} \dot{x}(s) \mathrm{d} s-B \dot{x}(t)\right] . }
\end{aligned}
$$

Then, we have

$$
\dot{V}(x(t)) \leqslant \mathrm{e}^{2 \alpha t}\left[\xi^{\mathrm{T}}(t) \Omega \xi(t)\right]
$$

where $\xi(t)=\left[\begin{array}{lll}x^{\mathrm{T}}(t) & x^{\mathrm{T}}\left(t-h_{a}\right) & \dot{x}^{\mathrm{T}}(t)\end{array}\right]^{\mathrm{T}}$. Consequently, condition (6) is sufficient to obtain $\dot{V}(x(t))<0$ ensuring the $\alpha$-asymptotic stability of the positive system (5) with varying-time delay.

\section{References}

[1] W. R. Zhao. Global exponential stability analysis of CohenGrossberg neural network with delays. Communications in Nonlinear Science and Numerical Simulation, vol. 13, no. 5, pp. $847-856,2008$

[2] J. H. Kim. Note on stability of linear systems with timevarying delay. Automatica, vol. 47, no. 9, pp. 2118-2121, 2011.

[3] J. J. Batzel, F. Kappel. Time delay in physiological systems: Analyzing and modeling its impact. Mathematical Biosciences, vol. 234, no. 2, pp. 61-74, 2011.

[4] P. Ramachandran, Y. M. Ram. Stability boundaries of mechanical controlled system with time delay. Mechanical Systems and Signal Processing, vol. 27, no. 2, pp. 523-533, 2012.

[5] K. Q. Gu, S. I. Niculescu. Survey on recent results in the stability and control of time-delay systems. Journal of Dynamic Systems, Measurement and Control, vol. 125, no. 2, pp. 158-165, 2003

[6] X. L. Zhu, G. H. Yang. New results of stability analysis for systems with time-varying delay. International Journal of
Robust and Nonlinear Control, vol. 20, no. 5, pp. 596-606, 2010

[7] M. J. Park, O. M. Kwon, J. H. Park, S. M. Lee. Simplified stability criteria for fuzzy Markovian jumping Hopfield neural networks of neutral type with interval time-varying delays. Expert Systems with Applications, vol. 39, no. 5, pp. 5625-5633, 2012.

[8] W. Elloumi, W. Kacem, M. Chaabane, D. Mehdi. Exponential stability criteria for systems with time-varying delays In Proceedings of the 2nd International Conference on Systems and Control, IEEE, Marrakesh, Morocco, pp. 105-109, 2012 .

[9] L. Farina, S. Rinaldi. Positive Linear Systems: Theory and Applications, New York: Wiley, 2000.

[10] T. Kaczorek. Positive $1 D$ and $2 D$ Systems, New York: Springer-Verlag, 2001.

[11] M. Ait Rami, F. Tadeo. Positive observation problem for linear time-lag positive systems. In Proceedings of the 3rd IFAC Symposium on Power System, Structure and Control, Mabu Thermas Convention Center, Brazil, pp. 536$541,2007$.

[12] S. Q. Zhu, M. Meng, C. H. Zhang. Exponential stability for positive systems with bounded time-varying delays and static output feedback stabilization. Journal of the Franklin Institute, vol. 350, no. 3, pp. 617-636, 2013.

[13] S. Oucheriah. Synthesis of controllers for time-delay systems subject to actuator saturation and disturbance. Journal of Dynamic Systems, Measurement and Control, vol. 125, no. 2, pp. 244-249, 2003.

[14] A. Benzaouia, A. Hmamed, F. Tadeo. Stabilization of controlled positive delayed continuous-time systems. International Journal of Systems Sciences, vol. 41, no. 12, pp. 1473$1479,2010$.

[15] A. Benzaouia, A. El Hajjaji. Delay-dependent stabilization conditions of controlled positive T-S fuzzy systems with time varying. International Journal of Computing Innovation and Control, vol. 7, no. 4, pp. 1533-1548, 2011.

[16] A. Benzaouia. Saturated Switching Systems, London: Springer, 2012.

[17] M. Nachidi, F. Tadeo, A. Benzaouia, M. Ait Rami. Static output-feedback for Takagi-Sugeno systems with delays. International Journal of Adaptive Control and Signal Processing, vol. 25, no. 4, pp. 295-312, 2011.

[18] A. Hmamed, M. Ait Rami, A. Benzaouia, F. Tadeo. Stabilization under constrained states and controls of positive systems with time delays. European Journal of Control, vol. 18 , no. 2 , pp. $182-190,2012$

[19] T. Kaczorek. Realization problem for positive linear systems with time delay. Mathematical Problems in Engineering, vol. 4, no. 4, pp. 455-463, 2005.

[20] G. M. Xie, L. Wang. Reachability and controllability of positive linear discrete-time systems with time-delays. In Proceedings of the 1st Multidisciplinary International Symposium on Positive Systems: Theory and Applications, Lecture Notes in Control and Information Science, Springer Verlag: Rome, Italy, pp. 377-384, 2003. 
[21] M. Bolajraf. Robust Control and Estimation for Positive Sstems, Ph. D. dissertation, University of Valladolid, Spain, 2012 .

[22] V. N. Phat, Y. Khongthamb, K. Ratchagit. LMI approach to exponential stability of linear systems with interval timevarying delays. Linear Algebra and Applications, vol. 436, no. 1, pp. 243-251, 2011

[23] T. Botmart, P. Niamsup. Robust exponential stability and stabilizability of linear parameter dependent systems with delays. Applied Mathematics and Computation, vol. 217, no. 6, pp. 2551-2566, 2010.

[24] V. Chellaboina, W. M. Haddad, J. Ramakrishnan, J. M Bailey. On monotonicity of solutions of nonnegative and compartmental dynamical systems with time delays. In Proceedings of the 42nd Conference on Decision and Control, IEEE, Hawaii, USA, pp. 4008-4013, 2003.

[25] M. Araki. Application of $M$-matrices to the stability problems of composite dynamical systems. Journal of Mathematical Analysis and Applications, vol.52, no. 2, pp.309321, 1975.

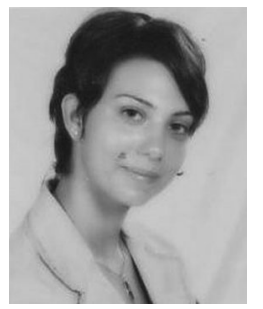

Wafa Elloumi received the master degrees in automatic control and industrial computing from the University of Sfax, Tunisia in 2012. Currently, she is a Ph. D. candidate in electrical engineering at the National Engineering School of Sfax (ENIS), Tunisia, and an associate researcher in the Laboratory of Sciences and Techniques of Automatic \& Computer Engineering Lab-STA, University of Sfax.

Her research interests include analyzing of stability and stabilization of continuous delayed systems as well as positive systems.
E-mail: elloumi.wafa@gmail.com

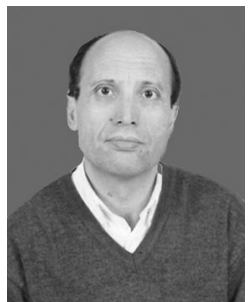

Abdellah Benzaouia received the degree of electrical engineering at the Mohammedia school (Rabat) in 1979 and the Doctorat Ph. D. at the University Cadi Ayyad in 1988 . He is actually professor at the University of Cadi Ayyad (Marrakech) where he is also the head of the Automatic Control Laboratory of Environment and Process Transfers, , affiliated to National Center for Scientific and Technical Research. He collaborates with many teams in France, Canada, Spain and Italy.

His research interests include control, robust control, pole assignment, systems with Markovian jumping parameters, hybrid systems and fuzzy systems.

E-mail: benzaouia@uca.ca (Corresponding author)

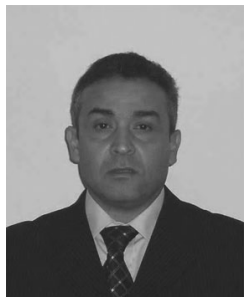

Mohamed Chaabane received his doctorate degree in electrical engineering from the University of Nancy, France in 1991. He was associate professor at the University of Nancy and was a researcher at Center of Automatic Control of Nancy (CRAN) from 1988-1992. Since 1997, he is holding a research position at Laboratory of Sciences and Techniques of Automatic control \& Computer Engineering (Lab-STA), the National Engineering School of Sfax (ENIS), Tunisia. Currently, he is a professor in automatic control at ENIS, Currently, he is an associate editor of International Journal on Sciences and Techniques of Automatic Control \& Computer Engineering (www.statn.com), Tunisia. His research interests include robust and optimal control, delay systems, and descriptor systems.

E-mall: chaabane.ucpi@gmail.com 